Milad Torabfam and Hoda Jafarizadeh-Malmiri*

\title{
Microwave-enhanced silver nanoparticle synthesis using chitosan biopolymer: optimization of the process conditions and evaluation of their characteristics
}

https://doi.org/10.1515/gps-2017-0139

Received September 9, 2017; accepted October 17, 2017; previously published online December 4, 2017

Abstract: A facile and green synthesis of silver nanoparticles (AgNPs) by aqueous chitosan solution and microwave irradiation is proposed as a cost effective and environmentally benevolent alternative to chemical and physical methods. With this aim, different amounts of chitosan solution (3-9 ml) with several concentration $(4-6 \% \mathrm{w} / \mathrm{v})$ and $3 \mathrm{ml}$ of the silver salt solution $(0.5 \% \mathrm{w} / \mathrm{v})$ were mixed and microwave irradiated for $100 \mathrm{~s}$. Response surface methodology (RSM) was used to evaluate the effects of the amount and concentration of chitosan solution on the particle size and concentration of the synthesized AgNPs. The optimum AgNPs synthesis process was obtained using $9 \mathrm{ml}$ of $0.4 \%$ (w/v) chitosan solution. The spherical and more stable AgNPs synthesized at optimum conditions had particle size, concentration, polydispersity index (PDI) and zeta potential values of $37 \mathrm{~nm}$, $69 \mathrm{ppm}, 0.557$ and $+50 \mathrm{mV}$, respectively. The synthesized AgNPs indicated strong antifungal activity toward Aspergillus flavus and high antibacterial activity against both Gram-positive (Staphylococcus aureus) and Gram-negative bacteria (Escherichia coli).

Keywords: antimicrobial activity; chitosan; microwave irradiation; optimization; silver nanoparticles.

\section{Introduction}

Synthesis and application of nanoscale materials has emerged as one of the most intensively studied areas in nanoscience and nanotechnology in recent years, due to the significant degree of difference in their physico-chemical and biological properties as compared to macroscale $[1,2]$. Interest in metal nanoparticles (NPs) as antimicrobial agents has increased during the last decade. Among

\footnotetext{
*Corresponding author: Hoda Jafarizadeh-Malmiri, Faculty of Chemical Engineering, Sahand University of Technology, 51335-1996 Sahand, Tabriz, Iran, e-mail: h_jafarizadeh@sut.ac.ir Milad Torabfam: Faculty of Chemical Engineering, Sahand University of Technology, 51335-1996 Sahand, Tabriz, Iran
}

the noble metal NPs, silver NPs (AgNPs) have indicated the highest antibacterial, antifungal and antiviral activities toward numerous species of microorganisms [3, 4]. In fact, Ag NPs can be easily attached to the microorganism's membrane, change its permeability and cause cell death [5].

Several physical and chemical methods have been developed for formation of AgNPs [6-8]. In the chemical reduction method, as a most popular technique in NPs synthesis, fabricated AgNPs have been achieved in aqueous solution by the reduction of silver ions using chemical compounds such as sodium borohydride. Furthermore, in order to avoid agglomeration of AgNPs, stabilizer agents such as synthetic polymers (i.e. polyvinylpyrrolidone, polyethylene glycol and polyacrylamide) have been added into the solution [6]. Due to non-biodegradability and toxicity of the chemical reducing and stabilizing compounds used in the chemical reduction method, the utilization of the resulting colloidal solution of the AgNPs drastically decreases, especially in medical applications $[1,3]$.

Several studies have been successfully done on "green" synthesis of colloidal AgNPs using natural polymers such as polysaccharides (i.e. chitosan, guar gum and Arabic gum) [6, 9, 10]. Among the natural polysaccharides, chitosan is very attractive for use as both a reducing and a stabilizing agent in the fabrication of AgNPs $[1,2,11]$. Chitosan is a cationic renewable polymer with a number of functional groups in its structure such as $-\mathrm{NH}_{2}$ and - $\mathrm{OH}$. Hydroxyl groups of chitosan strongly participate in reducing silver ions and converting them into silver elements and AgNPs, and its primary amine group has a strong effect on the stabilizing of the formed NPs. Furthermore, due to high antimicrobial properties of chitosan, it is expected that it in the synthesis of AgNPs colloidal solutions can result in a synergy of their antiseptic properties for potential biomedical applications [6, 12, 13]. Several studies have been done on synthesis of AgNPs using chitosan $[2,7,14,15]$. However, numerous researches have indicated that the rate of synthesis of AgNPs using chitosan could be increased drastically in combination with different heating methods such as gamma ray irradiation [1,9], ultrasound [6], autoclave [15] and microwave irradiation [13]. 
Microwave-assisted green synthesis of metal NPs in solution has been getting much more attention in recent years due to its short reaction time, low energy consumption and better product yield [16]. As compared to other heating methods, microwave heating offers fast uniform nucleation and growth of NPs during the reaction, which in turn leads to fabrication of NPs with different shapes, high concentrations and more uniform size distributions $[17,18]$. Several studies have been completed based on green synthesis of AgNPs and microwave heating using pectin [19], Eucalyptus globulus leaf extract [20], Rhizome extract of Alpinia galanga [21] and Fraxinus excelsior leaf extract [22].

The main objectives of the present study were: (i) to use microwave radiation to accelerate nucleation and synthesis of AgNPs, (ii) to optimize the amount and concentration of chitosan solution to fabricate stabilized AgNPs with smaller particle size and polydispersity index (PDI), and high concentration, and (iii) to evaluate antifungal and antibacterial activities of the synthesized AgNPs colloid against several microorganisms.

\section{Materials and methods}

\subsection{Materials}

Chitosan with medium molecular weight as both reducing and stabilizing agent was obtained from Aldrich (Aldrich Co. Steinheim, Germany). Silver nitrate $\left(\mathrm{AgNO}_{3}\right)$ as silver ions precursor was provided from Dr. Mojallali (Dr. Mojallali Chemical Complex Co., Tehran, Iran). Standard AgNPs solution, concentration of $1000 \mathrm{ppm}$ and particle size of $10 \mathrm{~nm}$, was purchased from Tecnan-Nanomat (Navarra, Spain).

Aspergillus flavus (PTCC 5004), Escherichia coli (PTCC 1395) and Staphylococcus aureus (PTCC 1189) were obtained from microbial Persian type culture collection (Tehran, Iran). Potato dextrose agar (PDA) and nutrient agar as culture media were provided from Oxoid (Oxoid Ltd., Hampshire, England) and Biolife (Biolife Co. Milan, Italy), respectively. Deionized double distilled water was used to prepare all the aqueous solutions.

\subsection{Chitosan solution preparation and its use in synthesis of AgNPs}

According to the literature, chitosan solutions with different concentrations $(4-6 \% \mathrm{w} / \mathrm{v})$ were prepared by dissolving $4-6 \mathrm{~g}$ of chitosan in $100 \mathrm{ml}$ of the $1 \%$ acetic acid solution $[6,7,10]$. Different amounts of the chitosan solutions (3-9 ml) were then mixed with $3 \mathrm{ml}$ of the silver salt solution ( $0.5 \mathrm{~g} \mathrm{AgNO}_{3}$ in $100 \mathrm{ml}$ deionized water) and the mixture solutions were exposed to microwave irradiation using a microwave oven (MG-2312W, LG Co., Seoul, South Korea) at a constant power of $800 \mathrm{~W}$ and constant microwave exposure times (100 s).

\subsection{Analysis}

2.3.1 Surface plasmon resonance: Due to surface plasmon resonance (SPR) of the synthesized AgNPs, their formation can be easily monitored and recorded by scanning the mixture solutions under a spectrophotometer. A UV-Vis spectrophotometer (Jenway UV-Vis spectrophotometer 6705, UK) was used to characterize the AgNPs solution SPR. In fact, due to the excitation of surface plasmon vibration bands, the color of the reaction solution is changed and broad emission peaks $\left(\lambda_{\max }\right)$ are centered $(380-450 \mathrm{~nm})$ [4].

2.3.2 Fourier transform-infrared spectra: In order to evaluate of the reducing and stabilizing potential of chitosan to synthesis AgNPs, Fourier transform infrared (FT-IR) spectra of chitosan containing AgNPs and pure chitosan were monitored in $\mathrm{KBr}$ pellets using an FT-IR spectrophotometer (Bruker Optics, Germany). The range 4000$400 \mathrm{~cm}^{-1}$ was used to record the FT-IR spectra [23].

2.3.3 Formed AgNPs characteristics: Concentration of the synthesized AgNPs can be easily estimated using UV-Vis spectroscopy and based on the standard curve (equation), which was fitted and generated using serial dilute solutions of AgNPs (10-1000 ppm). This curve correlated the absorbance of the formed AgNPs to their concentration. Particle size, PDI, particle size distribution and zeta potential values of the synthesized AgNPs were measured using a dynamic light scattering particle size analyzer (Nanotrac Wave, Microtrac, USA). To study the morphology of the synthesized AgNPs, including their shape and size, a transmission electron microscope (CM120, Philips, Amsterdam, The Netherlands) with an acceleration voltage of $120 \mathrm{kV}$ was used.

2.3.4 Antimicrobial assay: The well diffusion method was carried out to examine antibacterial activities of the synthesized AgNPs. In fact, $0.1 \mathrm{ml}$ samples of the provided bacterial suspensions adjusted to 0.5 McFarland standard, containing $1.5 \times 10^{8}$ colony forming units of bacteria in $1 \mathrm{ml}$ of prepared suspensions, were inoculated on nutrient agar culture media at the plates ( $90 \mathrm{ml}$ in diameter) and then some wells (with diameter of $5 \mathrm{~mm}$ ) were created in the inoculated culture media. Some $10 \mu \mathrm{l}$ of the synthesized AgNPs solutions were poured in each hole. The provided plates were then placed in the incubator at $37^{\circ} \mathrm{C}$ for $24 \mathrm{~h}$. The antifungal activity of the fabricated AgNPs was evaluated using a method based on radial mycelia growth of Aspergillus flavus inhibition on the culture media containing PDA incorporated with AgNPs [4]. A pure culture of the A. flavus was isolated from a $5 \mathrm{~mm}$ in diameter agar disk and located in the center of a plate containing PDA, as the control sample, and plates containing PDA and chitosan, and PDA and chitosan amended with AgNPs. The provided plates were then incubated at ambient temperature $26 \pm 2^{\circ} \mathrm{C}$ for 1 week. Mycelia growth diameters of the fungus on the plates were measured daily during 7 days of incubation, and antifungal activity of the synthesized AgNPs was stated as inhibition of the fungal hyphae growth (mm).

2.3.5 Design of experiments, statistical analysis and optimization procedure: Based on central composite design and two independent variables (synthesis parameters), namely concentration of chitosan solution ( $0.25-1 \% \mathrm{w} / \mathrm{v})\left(\mathrm{X}_{1}\right)$ and amount of chitosan solution (3-9 ml) $\left(\mathrm{X}_{2}\right), 13$ experiment runs, including four factorial points, four star points and five central points were generated (Table 1). Response surface methodology (RSM) based on a second-order polynomial 
Table 1: Central composite design (CCD) and response variables for synthesis of silver nanoparticles (AgNPs).

\begin{tabular}{|c|c|c|c|c|c|c|}
\hline \multirow{2}{*}{$\begin{array}{l}\text { Sample } \\
\text { number }\end{array}$} & \multirow{2}{*}{$\begin{array}{r}\text { Concentration of } \\
\text { chitosan solution }(w / v)\end{array}$} & \multirow{2}{*}{$\begin{array}{r}\text { Amount of chitosan } \\
\text { solution }(\mathrm{ml})\end{array}$} & \multirow{2}{*}{$\frac{\lambda_{\text {max }}}{\text { Experimental }}$} & \multirow{2}{*}{$\frac{(\mathrm{nm})}{\text { Predicted }}$} & \multirow{2}{*}{$\frac{\text { Concentration }}{\text { Experimental }}$} & \multirow{2}{*}{$\frac{(\mathrm{ppm})}{\text { Predicted }}$} \\
\hline & & & & & & \\
\hline 1 & 0.500 & 6.00 & 415 & 415 & 33 & 31 \\
\hline 2 & 0.500 & 6.00 & 414 & 415 & 28 & 31 \\
\hline 3 & 0.500 & 6.00 & 416 & 415 & 32 & 31 \\
\hline 4 & 0.400 & 6.00 & 405 & 407 & 22 & 26 \\
\hline 5 & 0.570 & 8.12 & 418 & 418 & 76 & 78 \\
\hline 6 & 0.500 & 6.00 & 414 & 415 & 34 & 31 \\
\hline 7 & 0.500 & 6.00 & 416 & 415 & 27 & 31 \\
\hline 8 & 0.500 & 3.00 & 401 & 402 & 19 & 20 \\
\hline 9 & 0.429 & 8.12 & 417 & 415 & 56 & 54 \\
\hline 10 & 0.570 & 3.87 & 406 & 405 & 27 & 29 \\
\hline 11 & 0.500 & 9.00 & 419 & 420 & 78 & 80 \\
\hline 12 & 0.600 & 6.00 & 408 & 410 & 53 & 51 \\
\hline 13 & 0.429 & 3.87 & 404 & 402 & 21 & 18 \\
\hline
\end{tabular}

equation [Eq. (1)] was used to study the main, quadratic and interaction effects of synthesis parameters on the response variable, namely, broad emission peak $\left(\lambda_{\max }, \mathrm{nm}\right)\left(\mathrm{Y}_{1}\right)$ and concentration $(\mathrm{ppm})$ $\left(\mathrm{Y}_{2}\right)$ of the synthesized AgNPs. RSM has several advantages including creating numerous valuable data using a small run of experiments and the ability of estimating the independent variables interactions on the response variables [4]. The $\lambda_{\max }$ could be related to the particle size of the fabricated NPs as the longer wavelengths correspond to increase in particle size [3, 4].

$$
Y=\beta_{0}+\beta_{1} X_{1}+\beta_{2} X_{2}+\beta_{11} X_{1}^{2}+\beta_{22} X_{2}^{2}+\beta_{12} X_{1} X_{2}
$$

In Eq. (1), $Y$ is the response variable, $X_{1}$ and $X_{2}$ are synthesis parameters, $\beta_{0}$ is a constant, $\beta_{1}$ and $\beta_{2}$ correspond to the linear terms, $\beta_{11}, \beta_{22}$ represent the quadratic terms and $\beta_{12}$ indicates the interaction terms. The fitness of the obtained model was evaluated using the coefficient of determination $\left(R^{2}\right)$, adjusted coefficient of determination $\left(R^{2}\right.$-adj) and lack of fit (p-value) [24, 25]. Significance determinations of the resulted models were studied using analysis of variance in terms of $\mathrm{p}$-value and $\mathrm{F}$ ratio. In fact, lower $\mathrm{p}$-values $(\mathrm{p}<0.05)$ and high values of $\mathrm{F}$ ratio were considered as statistically significant [26]. In order to obtain the optimum levels of independent synthesized variables with the desired response variables (minimum particle size, $\lambda_{\max }$, and maximum concentration of the synthesized AgNPs) numerical response optimization and graphical optimization using a two dimensional contour plot were used. Three additional approval tests were performed at obtained optimum synthesis conditions to verify the obtained results. Central composite design, RSM, analysis of variance and optimization processes were run using the software Minitab v.16 statistical package (Minitab Inc., PA, USA).

\section{Results and discussion}

\subsection{Formation of AgNPs}

Metal NPs have free electrons, which cause an SPR absorption band. Due to the excitation of surface plasmon vibration bands, broad emission peaks $\left(\lambda_{\max }\right)$ can be observed for AgNPs between $380 \mathrm{~nm}$ and $450 \mathrm{~nm}$ wavelength and this causes the changes in the color of the synthesized AgNPs solution [3, 4, 23]. By the formation of the AgNPs, the solution color turned to yellow-brown. The yellow-brown color of the mixture solutions, after exposure to microwave irradiation, was related to the SPR and indicated that AgNPs were formed (Figure 1). As clearly observed in Figure $2, \lambda_{\max }$ of the synthesized AgNPs at center point synthesized conditions was obtained at $415 \mathrm{~nm}$ which was in the favorable range for AgNPs. This indicated that chitosan solution successfully reduced silver ions and formed AgNPs. Figure 3 shows the FT-IR spectra of pure chitosan and chitosan containing AgNPs solutions. The IR spectrum of chitosan absorption bands at $3453.67 \mathrm{~cm}^{-1}$ and $2073.08 \mathrm{~cm}^{-1}$ represent the $-\mathrm{NH}_{2},-\mathrm{OH}$ and $\mathrm{N}=\mathrm{C}$ groups. Absorption bands at $1638.06 \mathrm{~cm}^{-1}$ and $1422 \mathrm{~cm}^{-1}$ are related to the amino group bending vibrations and the $-\mathrm{OH}$ group of the primary alcoholic group, respectively. The amino group has a characteristic absorption band in the region of $3400-3500 \mathrm{~cm}^{-1}$. The absorption
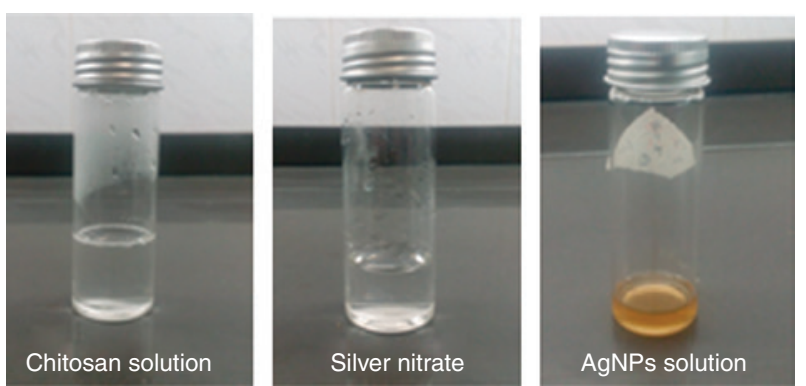

Figure 1: Color and appearance of the chitosan, silver nitrate and mixture solution containing silver nanoparticles (AgNPs). 


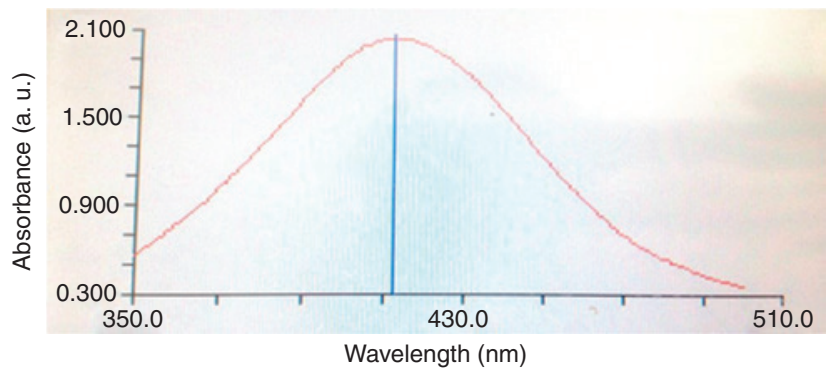

Figure 2: Surface plasmon resonance spectrum of the synthesized silver nanoparticles (AgNPs) at center point synthesis conditions.

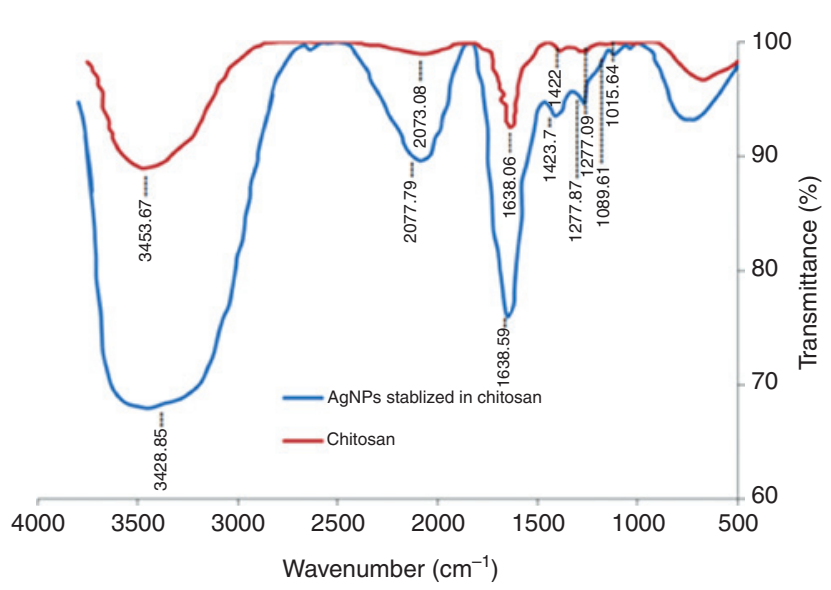

Figure 3: Fourier transform infrared (FT-IR) spectrum of chitosan and chitosan containing silver nanoparticles (AgNPs).

band at $1277.09 \mathrm{~cm}^{-1}$ and $1015.64 \mathrm{~cm}^{-1}$ is attributed to the $-\mathrm{CH}_{2} \mathrm{X}$ alkyl group of chitosan. The FT-IR spectrum of chitosan containing AgNPs is the same as the FT-IR spectrum of pure chitosan and the differences in intensity of peak indicate synthesis of AgNPs by chitosan. The variation in the shape and peak positions of the $-\mathrm{NH}_{2}$ and $-\mathrm{OH}$ at $3428.85 \mathrm{~cm}^{-1}$ occurred because of contribution toward the reduction and stabilization process.

\subsection{Models generation}

According to the experimental data obtained for the response variables (Table 1), final models were generated. The predictable regression coefficients and the terms related to the accuracy $\left(R^{2}, R^{2}\right.$-adj and lack of fit) of the models are given in Table 2. $\mathrm{F}$ ratio and $\mathrm{p}$-values of all terms in the obtained models are also shown in Table 3. The quite high values for $\mathrm{R}^{2}$ and $\mathrm{R}^{2}$-adj of $\lambda_{\max }(0.9584$ and 0.9237 , respectively) and concentration ( 0.9830 and 96.88 , respectively) of the synthesized AgNPs confirmed the suitability of the resulting models. Furthermore, attained
Table 2: Regression coefficients, $R^{2}$, adjusted $R^{2}\left(R^{2}\right.$-adj) and probability values for the generated models.

\begin{tabular}{lrr}
\hline Regression coefficient & $\boldsymbol{\lambda}_{\text {max }}(\mathbf{n m})$ & Concentration $(\mathbf{p p m})$ \\
\hline$\beta_{0}$ (constant) & 222.793 & 245.487 \\
$\beta_{1}$ (main effect) & 603.306 & -781.500 \\
$\beta_{2}$ (main effect) & 9.042 & -26.775 \\
$\beta_{11}$ (quadratic effect) & -571.967 & 775.375 \\
$\beta_{22}$ (quadratic effect) & -0.436 & 2.163 \\
$\beta_{12}$ (interaction effect) & -1.667 & 21.630 \\
$\mathrm{R}^{2}$ & $95.84 \%$ & $98.30 \%$ \\
$\mathrm{R}^{2}$-adj & $92.37 \%$ & $96.88 \%$ \\
Lack of fit & 1.724 & 3.751 \\
$\mathrm{p}$-Value (regression) & 0.000 & 0.000 \\
\hline
\end{tabular}

$\beta_{0}$ is a constant, $\beta_{\mathrm{i}}, \beta_{\mathrm{ii}}$ and $\beta_{\mathrm{ij}}$ are the linear, quadratic and interaction coefficients of the quadratic polynomial equation, respectively.

1 , Concentration of chitosan solution (\% w/v); 2 , amount of chitosan solution (ml).

Table 3: Significance probability ( $p$-value, $F$ ratio) of regression coefficients in the second-order polynomial models.

\begin{tabular}{|c|c|c|c|c|c|}
\hline & \multicolumn{2}{|c|}{ Main effects } & \multicolumn{2}{|c|}{$\begin{array}{r}\text { Quadratic } \\
\text { effects }\end{array}$} & \multirow{2}{*}{$\begin{array}{r}\begin{array}{r}\text { Interaction } \\
\text { effects }\end{array} \\
\mathrm{X}_{12}\end{array}$} \\
\hline & $\mathrm{X}_{1}$ & $x_{2}$ & $\mathrm{X}_{1}^{2}$ & $x_{2}^{2}$ & \\
\hline \multicolumn{6}{|c|}{$\lambda_{\max }\left(\mathrm{Y}_{1}, \mathrm{~nm}\right)$} \\
\hline $\mathrm{p}$-Value & 0.011 & 0.038 & 0.014 & 0.027 & 0.782 \\
\hline Fvalue & 12.96 & 7.04 & 11.58 & 8.41 & 0.08 \\
\hline \multicolumn{6}{|c|}{ Concentration $\left(\mathrm{Y}_{2}, \mathrm{ppm}\right)$} \\
\hline p-Value & 0.044 & 0.011 & 0.040 & 0.001 & 0.134 \\
\hline F value & 6.50 & 13.00 & 6.38 & 43.09 & 2.99 \\
\hline
\end{tabular}

1 , Concentration of chitosan solution ( $\% \mathrm{w} / \mathrm{v}) ; 2$, amount of chitosan solution (ml).

non-significant lack of fits for the models $(p>0.05)$ approved its adequate fitness to the synthesis parameter effects (Table 2). As clearly observed in Table 3, the main and quadratic effects of synthesized parameters, concentration of chitosan solution $\left(\mathrm{X}_{1}\right)$ and amount of chitosan solution $\left(\mathrm{X}_{2}\right)$ had significant $(\mathrm{p}<0.05)$ effects on both $\lambda_{\max }$ (particle size) and concentration of the formed AgNPs, while their interaction had non-significant effects on the response variables.

\subsection{Effect of the synthesized parameters on $\lambda_{\max }$ of the formed AgNPs}

The experimental value of the $\lambda_{\max }$ for the synthesized AgNPs changed between $401 \mathrm{~nm}$ and $419 \mathrm{~nm}$ (Table 1), which was in a favorable range for AgNPs. The particle 
size of the synthesized AgNPs could be correlated with their $\lambda_{\max }$, where longer wavelengths in $\lambda_{\text {max }}$ of AgNPs was associated to their bigger size [4]. The obtained results illustrated that AgNPs could be easily synthesized using natural reductants existing in chitosan solution without applying other chemical-reducing agents. Figure 4 indicates the effects of concentration and amount of chitosan solution on the $\lambda_{\max }$ of the synthesized AgNPs. As clearly observed in Figure 4, minimum particle size $\left(\lambda_{\max }\right)$ was obtained at both minimum concentration and amount of chitosan solution, and maximum particle size was obtained at maximum amount of chitosan solution. The obtained result can be explained by the fact that at constant concentration of chitosan solution, by increasing the amount of chitosan solution, the concentration of reducing agents and volume of the mixture solution increased, which in turn increased the opportunities of the nucleation and growth of the AgNPs [27]. The obtained results indicated that at a constant amount of chitosan, by increasing the chitosan concentration, the particle size of the formed AgNPs was decreased due to increasing the stabilizing potential of the mixture solution, which prevented the accumulation of the formed NPs. It seems that at high concentrations of chitosan, the hydroxyl group (band at $3447 \mathrm{~cm}^{-1}$ in Figure 3) formed a covering layer around the metal ions that prevented their agglomeration and enhanced the stability of the solution. The obtained results were in agreement with the finding of Venkatesham et al. [15]. They observed that the amine group in chitosan solution adsorbed strongly to silver metals and reduced the agglomeration of the synthesized NPs.

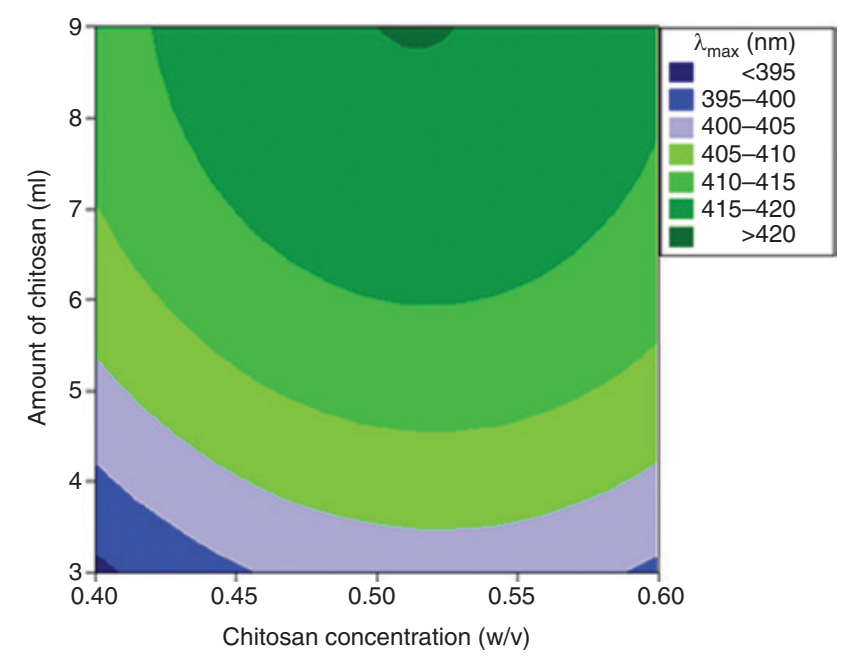

Figure 4: Contour plot for $\lambda_{\max }(\mathrm{nm})$ of the synthesized silver nanoparticles (AgNPs) solution as function of the amount and concentration of chitosan solution.

\subsection{Effect of the synthesized parameters on AgNPs concentration}

The experimental value of the concentration for the synthesized AgNPs ranged from $19 \mathrm{ppm}$ to $80 \mathrm{ppm}$ (Table 1). The effects of concentration and amount of chitosan solution on the concentration of the fabricated AgNPs are shown in Figure 5. As clearly observed in Figure 5, minimum AgNPs concentration was obtained at both minimum concentration and amount of chitosan solution, and maximum concentration was obtained at both maximum concentration and amount of chitosan solution. The results can be explained by the fact that at high concentration and amount of chitosan solution, the reducing potential of the synthesis solution containing $\mathrm{Ag}$ ions is higher than that of the synthesis solution based on the low concentration and amount of chitosan solution. By microwave exposing of such synthesis solution, the opportunities of the nucleation and growth of the AgNPs increased due to the increasing collision rate of the fast reduced $\mathrm{Ag}$ ions and preserve of the Ag elements in the synthesis solution [27]. According to Figure 5, the significant $(\mathrm{p}<0.05)$ effect of the amount of chitosan solution on the concentration of the AgNPs was higher than the second synthesis parameter, concentration of chitosan solution. The obtained result was reconfirmed by other results indicated in Table 3. As clearly observed in Table 3 , the lower p-value of the amount of chitosan solution as compared to the concentration of chitosan solution, indicated its higher significant $(\mathrm{p}<0.05)$ effect on the AgNPs concentration.

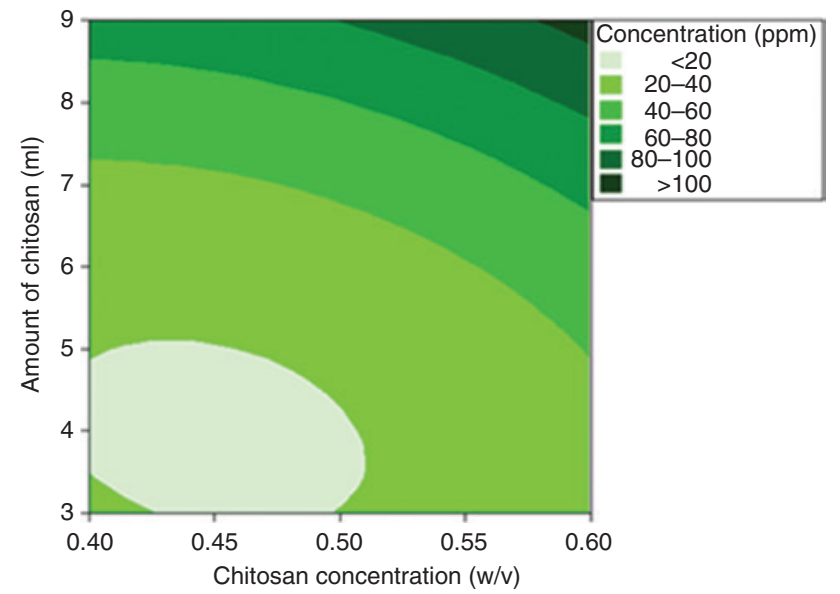

Figure 5: Contour plot for concentration (ppm) of the synthesized silver nanoparticles (AgNPs) solution as function of the amount and concentration of chitosan solution. 


\subsection{Optimization of the process for AgNPs synthesis}

The optimum process parameters for AgNPs synthesis would be attained when the formed AgNPs have the smallest mean particle size $\left(\lambda_{\max }\right)$ and highest concentration. The results of the numerical multiple optimizations demonstrated that the synthesized AgNPs obtained using $9 \mathrm{ml}$ of $0.4 \%$ (w/v) chitosan solution had minimum $\lambda_{\max }(412.6 \mathrm{~nm})$ and maximum concentration $(69 \mathrm{ppm})$. Graphical optimization based on an overlaid contour plot also indicated the optimum region for the synthesis parameters (Figure 6). The indicated white colored area in Figure 6 demonstrated the desired concentration and amount of chitosan solution to get the optimum AgNPs. In order to evaluate the adequacy of the obtained and fitted models by RSM, three AgNPs solutions were produced at the obtained optimal level of the synthesis conditions and were characterized in term of the $\lambda_{\max }$ and concentration. The measured experimental mean values for the $\lambda_{\max }$ and AgNPs concentration of these solutions were obtained at $412 \pm 1 \mathrm{~nm}$ and $72 \pm 4 \mathrm{ppm}$, respectively. Non-significant differences observed between the experimental and predicted values of the synthesized AgNPs in turn indicated the sufficiency of the generated models.

\subsection{Characteristics of the formed AgNPs at obtained optimum process parameters}

The results of the dynamic light scattering indicated that the synthesized AgNPs at obtained optimum synthesis conditions had mean particle size, PDI and zeta potential

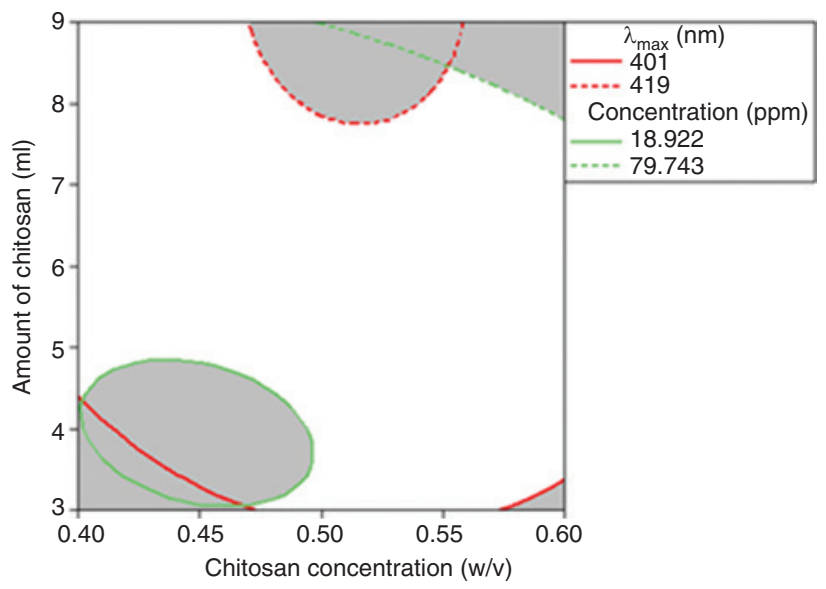

Figure 6: Overlaid contour plot of silver nanoparticles (AgNPs) $\lambda_{\max }$ and concentration with acceptable levels as function of amount and concentration of chitosan solution. values of $37 \mathrm{~nm}, 0.557$ and $+50 \mathrm{mV}$, respectively. As shown in Figure 7, the particle size distribution curve related to the synthesized AgNPs at optimum conditions indicated that all the particles are in the interval of $30-80 \mathrm{~nm}$. High zeta values of the formed AgNPs at obtained optimum process conditions indicated that the synthesized NPs had high stability. This result can be explained by the fact that the fabricated AgNPs were covered with a thin layer of the hydroxyl group (band at $3447 \mathrm{~cm}^{-1}$ in Figure 3) around the AgNPs and enhanced their stability in the solution. This layer contained the amino groups and the positive value of the zeta was related to the positive charges of those groups. The obtained results were in agreement with the finding of Yoksan and Chirachanchai [28]. They indicated that chitosan could be used to biosynthesize spherical AgNPs with a zeta potential value of $+40.4 \mathrm{mV}$. The transmission electron microscopy analysis indicated that the synthesized AgNPs were dispersed with spherical structures (Figure 8). This spherical shape indicated that the synthesized NPs had minimum surface energy and high

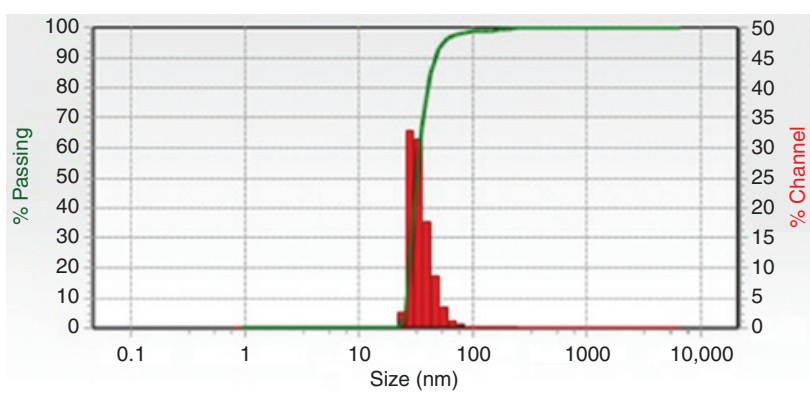

Figure 7: Particle size distribution of the synthesized silver nanoparticles (AgNPs) at obtained optimum synthesis conditions.

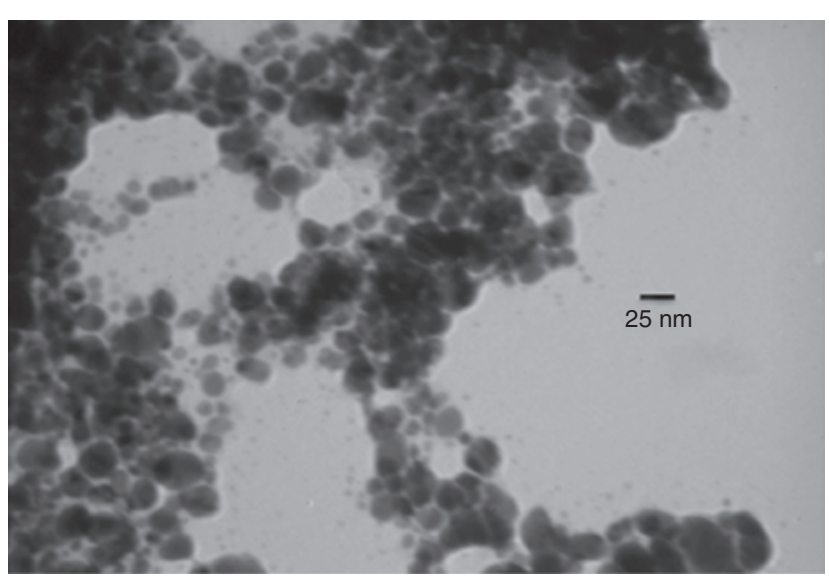

Figure 8: Transmission electron microscopy (TEM) image of the synthesized silver nanoparticles (AgNPs) at obtained optimum conditions. 


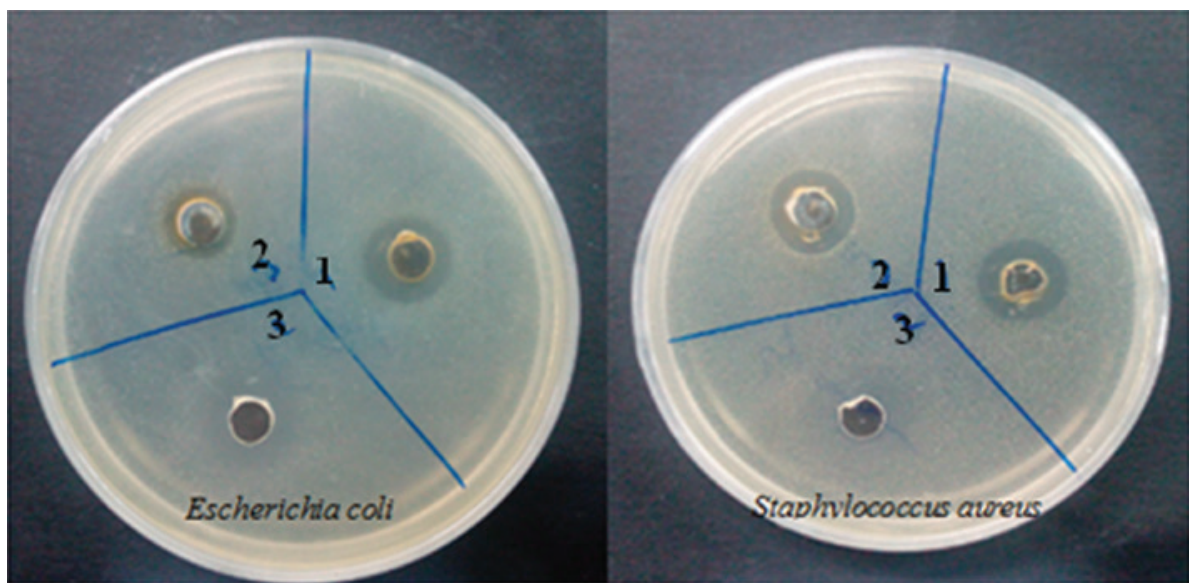

Figure 9: Created zones of inhibition with Staphylococcus aureus and Escherichia coli incubated at $37^{\circ} \mathrm{C}$ for $24 \mathrm{~h}$ for (1) chitosan solution containing silver nanoparticles (AgNPs), (2) chitosan solution and (3) distilled water.

thermodynamic stability, which confirmed the high value of the zeta potential of the synthesized AgNPs [3, 4].

\subsection{Antimicrobial activities of the formed AgNPs at obtained optimum process variables}

The antibacterial activity of synthesized AgNPs on growth of Gram-positive (S. aureus) and Gram-negative (E. coli) bacteria during incubation is shown in Figure 9. As shown in this Figure, the zones of inhibition for chitosan solution containing AgNPs (well number 1) were observed with S. aureus $(15 \mathrm{~mm})$ and E. coli $(14 \mathrm{~mm})$. Moreover, the clear zones for the chitosan solution (well number 2) were observed with $S$. aureus $(13 \mathrm{~mm})$ and E. coli $(12 \mathrm{~mm})$. Well number 3 containing distilled water did not show clear zones (Figure 9).

The antifungal activity of the fabricated AgNPs, as inhibitory effect on $A$. flavus hyphae growth, is shown in Figure 10. The results indicated that, compared to the control plates, the growth of $A$. flavus hyphae was drastically inhibited by the chitosan solution containing AgNPs and pure chitosan solution (Figure 10). The obtained results showed higher antifungal activity of the chitosan and formed AgNPs. This was in line with the findings of Roller and Covill [29] and Pulit et al. [30]. Roller and Covill [29] indicated that chitosan had a strong detrimental effect on the filamentous fungus and because of high antifungal properties they assessed the potential for using chitosan as a natural food preservative [29]. Pulit et al. [30] also indicated that synthesized AgNPs had a strong detrimental effect on the fungus Aspergillus niger. It was found that using nanosilver suspension at a concentration of $50 \mathrm{ppm}$ inhibited the growth of $A$. niger by $70 \%$. Filamentous fungi

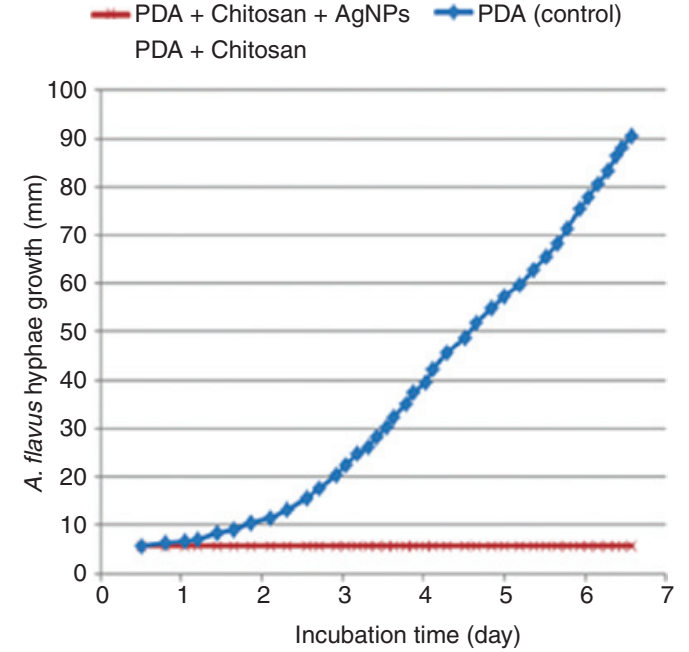

Figure 10: Antifungal effects of the synthesized silver nanoparticles (AgNPs) obtained at optimum conditions on Aspergillus flavus. Data are the mean value of three replicates.

such as A. flavus have also been successfully used to synthesize AgNPs. In our previous study, A. flavus was used in the synthesis of AgNPs [31]. Thereof, this fungus was chosen in the present study to evaluate the antifungal activity of the synthesized AgNPs.

\section{Conclusions}

Microwave irradiation is developed as an alternative energy source powerful enough to complete NPs synthesis in minutes, instead of hours or even days. The present work reports the facile, convenient and eco-friendly chitosan-mediated green synthesis of biopolymer conjugated 
AgNPs using microwaves. As compared to other polysaccharides and plant extract with high amounts of natural reducing and/or stabilizing biomolecules, chitosan as a natural biopolymer can be used as both a reducer and stabilizer in the synthesis of AgNPs and has unique properties including high antimicrobial activity and film forming. Finally, RSM was effectively used to enhance empirical models for the prediction of the concentration and particle size of the synthesized AgNPs solution, as a function of the concentration and amount of chitosan solution, under microwave irradiation.

Acknowledgments: The authors would like to acknowledge the Iran Nanotechnology Initiatives Council (INIC) for funding the development of an innovative methodology for safety assessment of industrial nanomaterials (grant no. 101470).

\section{References}

[1] Hettiarachchi MA, Wickramarachchi PASR. J. Sci. Univ. Kelaniya 2011, 6, 65-75.

[2] Ahmad MB, Tay MY, Shameli K, Hussein MZ, Lim JJ. Int. J. Mol. Sci. 2011, 12, 4872-4884.

[3] Mohammadlu M, Jafarizadeh-Malmiri H, Maghsoudi H. Int. Food Res. J. 2016, 23, 446-463.

[4] Mohammadlou M, Jafarizadeh-Malmiri H, Maghsoudi H. Green Process. Synth. 2017, 6, 31-42.

[5] Kim JS, Kuk E, Yu KN, Kim JH, Park SJ, Lee HJ, Kim SH, Park YK, Park YH, Hwang C. Biol. Med. 2007, 3, 95-101.

[6] Vega-Baudrit J, Alvarado-Meza R, Solera-Jiménez F. Av. Quím. 2014, 9, 125-129.

[7] Zain NM, Stapley AGF, Shama G. Carbohydr. Polym. 2014, 112, 195-202.

[8] Vivek R, Thangam R, Muthuchelian K, Gunasekaran P, Kaveri K, Kannan S. Process Biochem. 2012, 47, 2405-2410.

[9] Khodashenas B, Ghorbani HR. Arab. J. Chem. 2015, in press, doi:10.1016/j.arabjc. 2014.12.014.
[10] Yang C-H, Wangb L-S, Chen S-Y, Huang M-C, Li Y-H, Lin Y-C, Chen P-F, Shaw J-F, Huang K-S. Int. J. Pharm. 2016, 510, 493-500.

[11] Manikandan P, Sathiyabama M. J. Nanomed. Nanotechnol. 2015, 5, 251-255.

[12] Ghaz-Jahanian MA, Abbaspour-Aghdam F, Anarjan N, Berenjian A, Jafarizadeh-Malmir H. Mol. Biotechnol. 2015, 57, 201-218.

[13] Hiep NT, Khon HC, Niem VVT, Toi VV, Quyen TN, Hai ND, Anh MNT Int. J. Polym. Sci. 2016, doi.org/10.1155/2016/1584046.

[14] Modrzejewska Z, Zarzycki R, Sielski J. Prog. Chem. Appl. Chitin Deriv. 2010, XV, 63-72.

[15] Venkatesham M, Ayodhya D, Madhusudhan A, Babu NV, Veerabhadram G. Appl. Nanosci. 2014, 4, 113-119.

[16] Eskandari-Nojedehi M, Jafarizadeh-Malmiri H, RahbarShahrouzi J. Nanotechnol. Rev. 2016, 5, 537-548.

[17] Nadagouda MN, Speth TF, Varma RS. Acc. Chem. Res. 2011, 44, 469-478.

[18] Noroozi M, Zakaria A, Moksin MM, Wahab ZA, Abedini A. Int. J. Mol. Sci. 2012, 13, 8086-8096.

[19] Joseph S, Mathew B. Res. J. Recent Sci. 2014, 3, 185-191.

[20] Ali K, Ahmed B, Dwivedi S, Saquib Q, Al-Khedhairy AA, Musarrat J. PLoS One 2015, doi:10.1371/journal.pone.0131178.

[21] Joseph S, Mathew B. J. Nanopart. 2014, doi:10.1155/2014/967802.

[22] Parveen M, Ahmad F, Malla AM, Azaz S. Appl. Nanosci. 2016, 6, 267-276.

[23] Ahmadi 0, Jafarizadeh-Malmiri H, Jodeiri N. Green Process. Synth. 2018, 7, 231-240.

[24] Anarjan N, Jafarizadeh-Malmiri H, Nehdi IA, Sbihi HM, Al-Resayes SI, Tan CP. Int. J. Nanomed. 2015, 10, 1109-1118.

[25] Anarjan N, Jafarizadeh-Malmiri H, Ling TC, Tan CP. Int. J. Food Prop. 2014, 17, 937-947.

[26] Ahdno H, Jafarizadeh-Malmiri H. Food Bioprod. Process. 2017, 101, 193-204.

[27] Zhang Y, Cheng X, Zhang Y, Xue X, Fu Y. Colloids Surf. A. 2013, 423, 63-68.

[28] Yoksan R, Chirachanchai S. Mater. Sci. Eng. C. 2010, 30, 891-897.

[29] Roller S, Covill N. Int. J. Food Microbiol. 1999, 47, 67-77.

[30] Pulit J, Banach M, Szczygłowska R, Bryk M. Acta Biochim. Pol. 2013, 60, 795-798.

[31] Ghanbari S, Vaghari H, Sayyar Z, Adibpour M, JafarizadehMalmiri H. Green Process. Synth. 2018, 7, 217-224. 